\title{
PREVISIÓN DE NECESIDADES DE MANO DE OBRA DE CALIFICACIÓN ALTA. POSIBILIDAD DE APLICACIÓN DE LAS COMPARACIONES INTERNACIONALES AL CASO DE MÉXICO*
}

\author{
Michel Debeauvais \\ Centro de Desarrollo, OCDE
}

LA PUBlicación del libro Estructura ocupacional y educativa de la fuer$z a$ de trabajo $y$ niveles de desarrollo económico ${ }^{1}$ marca un avance importante en el trabajo de la OCDE sobre previsión de necesidades de mano de obra con miras a la planeación educativa.

La relación entre cambios en la estructura de la población activa y desarrollo económico ha sido objeto de verificación estadística sistemática en una comparación internacional que abarca 53 países, con base en el análisis de los censos de población de 1960.

El presente trabajo examina las conclusiones prácticas que pueden derivarse de éste desde el punto de vista de la planeación de los recursos humanos, tomando como ejemplo a México.

Los métodos de previsión de necesidades de mano de obra usados en el Proyecto Regional del Mediterráneo, y después en Perú y Argentina, consisten esencialmente en una secuencia de etapas a seguir para examinar sistemáticamente las relaciones entre la tasa de crecimiento económico, las metas de producción de los diferentes sectores económicos y, para cada uno de estos sectores, la evolución de la productividad, la estructura ocupacional y los niveles deseables de adiestramiento. Pero estas son relaciones de definición y no relaciones causales o funciones $;^{2}$ cuando estas tendencias tienen que expresarse cuantitativamente, los planificadores utilizan procedimientos cuyas desventajas reconocen ellos mismos:

* Trabajo presentado al Seminario Sobre Aplicación de Métodos de Previsión de la Oferta y la Demanda de Recursos Humanos de Nivel Superior, celebrado en El Colegio de México del 14 al 18 de diciembre de 1970. El título del original en inglés es: "Forecasting Highly Qualified Manpower Requirements. Applicability of International Comparison to the Case of Mexico". La traducción es de Daniel Murayama.

1 Occupational and Educational Structures of the Labour Force and Levels of Economic Development, OCDE, París, 1971, $321 \mathrm{pp}$.

2 Así, puede escribirse la siguiente fórmula para el último año de la previsión o para las tasas anuales de crecimiento:

$$
L_{i j k}=X \cdot \frac{X_{i}}{X} \cdot \frac{L_{i}}{X_{i}} \cdot \frac{L_{i j}}{L_{i}} \cdot \frac{L_{i j k}}{L_{i j}}
$$


1) Extrapolación de las tendencias pasadas (utilizada especialmente en Francia): este método supone que la productividad del trabajo, la estructura ocupacional y los perfiles de calificación varían en función del tiempo y por lo tanto independientemente de los factores económicos tales como niveles de producción, tasas de crecimiento económico, etc.

2) El ejemplo de las empresas más avanzadas, que son consideradas como modelos de todo un sector económico. Pero el período que debe transcurrir para "alcanzar" a esas empresas debe de escogerse más o menos arbitrariamente.

3) Comparaciones internacionales: en la mayoría de los casos, los planificadores han escogido en forma más o menos arbitraria como modelo a un país avanzado y un período para "alcanzar" al país escogido.

El estudio comparativo de la OCDE procura examinar todos los datos disponibles para estimar los coeficientes ocupacionales (y educativos) a la luz de indicadores económicos que representen en forma aproximada los respectivos niveles de desarrollo de los diferentes países y su progreso têcnico. El estudio trata de verificar si hay una relación directa entre todos los coeficientes ocupacionales y educativos (doce categorías ocupacionales y cuatro niveles de calificación) mediante cuatro indicadores económicos (producto por trabajador, consumo de energía por trabajador, inversión bruta acumulada durante siete años, un índice no monetario del desarrollo industrial) para la economía en su conjunto y para ocho ramas económicas. En esta publicación de la OCDE se presentan y discuten cientos de ecuaciones de regresión simple y múltiple. Los pocos resultados que aquí se mencionan pretenden ilustrar la manera en que podría utilizarse este tipo de análisis comparativo en la planificación de los recursos humanos en México.

En las diferentes técnicas de previsión de necesidades de mano de obra se usan más o menos explícitamente los siguientes supuestos:

1. El desarrollo económico (o el progreso técnico) demanda cambios en la estructura ocupacional de la fuerza de trabajo y en los niveles y tipos de calificación de las diferentes categorías ocupacionales. Estos cambios son relativamente más rápidos tratándose de categorías ocupacionales con una calificación alta (cuadros de nivel alto e intermedio) y entre éstos en lo que respecta a científicos y técnicos. Además, los niveles de estudio tienden a elevarse en las categorías ocupacionales. Por añadidura, la relación entre estas estructuras ocupacionales y educativas y el nivel de desarrollo económico se supone que es más estrecha en el caso de un sector económico que está directamente conectado con la producción que en el caso de servicios o de la economía en su conjunto. Las correlaciones deberían también elevarse cuando se examina una categoría ocupacional más homogénea, la cual esté más directamente relacionada con el proceso de producción.

2. Se supone que la situación de los diversos países en un momento

que muestra la naturaleza tautológica del método. De acuerdo con la notación usada en el trabajo de la OCDE, $L$ indica mano de obra, $X$ producto, $i$ los sectores económicos, $j$ las categorías ocupacionales y $k$ los niveles o tipos de calificación. 
dado es una aproximación aceptable de la evolución temporal del desarrollo de cada país en particular.

No sería difícil mostrar lo inadecuado de cada uno de estos supuestos, los cuales son simplificaciones de las relaciones entre la economía, el mercado de trabajo y el sistema educativo. Pero la verificación cuantitativa es un factor de juicio que no debe pasarse por alto; aunque no reemplace a la discusión teórica, un análisis de los datos disponibles puede arrojar luz sobre esto último.

Las ecuaciones de regresión simple usadas en el estudio de la OCDE comprenden tres índices significativos:

a) El coeficiente de regresión, el cual es aquí idéntico al coeficiente de elasticidad de la estructura ocupacional en relación con el producto por trabajador, ya que las ecuaciones se calcularon tomando los logaritmos de las dos variables; entre las funciones que se probaron, es ésta la que mejor se ajusta en casi todos los casos.

b) El coeficiente de correlación, cuyo cuadrado indica la proporción de varianza "explicada" por la variable independiente, que en todos los casos aquí presentados es el producto por trabajador. En el estudio de la OCDE también se utilizaron otros indicadores económicos: inversión por trabajador, consumo de energía por trabajador, un índice no monetario de desarrollo industrial, etc. En la mayoría de los casos, los resultados no difieren significativamente.

c) La desviación estándar del coeficiente de regresión, el cual no proporciona la misma información que el coeficiente de determinación, ya que el primero se calculó sólo para uno de los ejes (y en todos los casos se hace caso omiso de la desviación estándar del término constante). Esta desviación estándar permite determinar aproximadamente los límites de confianza del coeficiente de elasticidad, suponiendo que las desviaciones estén distribuidas "normalmente" a lo largo de la línea de regresión; se pasa también por alto el hecho de que se hayan tomado logaritmos de las variables, lo que implica una curva de desviación asimétrica para las variables que, en rigor, debería haberse tenido en cuenta.

La gráfica 1 muestra la relación observada en 43 países entre la proporción de trabajadores profesionales y técnicos (grupo 0 en el ISCO -Clasificación Internacional Estándar de Ocupaciones-) y producto por trabajador. La correlación es significativa en alto grado $\mathbf{y}$. explica el $83 \%$ de las diferencias entre varios países. México está muy cerca de la línea de regresión, con un porciento de trabajadores técnicos y profesionales (3.2\%) correspondiente a su nivel de desarrollo económico (Dls. 800 por trabajador).$^{3}$ El coeficiente de elasticidad de 0.66 , el cual ha sido verificado a nivel internacional, puede probablemente ser usado para las proyecciones. Extrapolando el crecimiento económico observado durante los años recientes $(6.8 \%$ en promedio al año entre 1959 y 1968) y considerando el aumento en la población activa (cerca de $3.3 \%$ al año), la ecuación de regresión muestra que la proporción de trabajadores técnicos y profesionales aumentaría

3 De acuerdo con series revisadas de las cuentas nacionales preparadas por el Banco de México, el PNB a precios de mercado sería de 150000 millones de pesos, esto es, Dls. 1060 por trabajador. No intentamos corregir las cifras del estudio de la OCDE de acuerdo con éstas. 
Profesionales y técnicos $(\mathrm{Lj} / \mathrm{L})$ y PROducto POR TRABajadoR $(\mathrm{X} / \mathrm{L})$ PARA LA ECONOMIA EN SU CONJUNTO

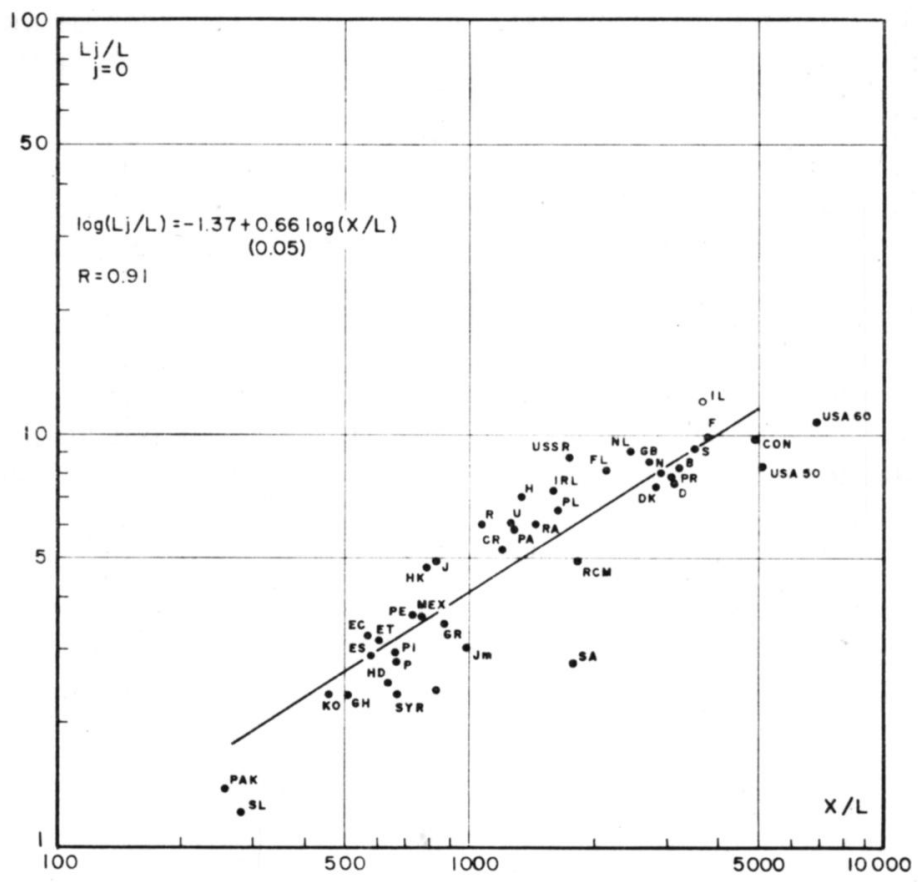

de $3.62 \%$ en 1960 a $4.54 \%$ en 1970 y $5.7 \%$ para 1980.4 Pero si aplicamos la prueba usual (con las reservas del caso ya mencionadas), los límites de confianza (a un nivel de 0.05 ) estarían entre $4.4 \%$ y $4.7 \%$ en 1970 y entre $5.35 \%$ a $6.1 \%$ en 1980 . Estos márgenes de incertidumbre son demasiado amplios para que el planificador los desprecie, ya que corresponderían en el caso de México a una probabilidad de error de 50000 trabajadores técnicos y profesionales en 1970 y de 160000 en 1980. Al emplear el coeficiente de elasticidad de 0.66 y la desviación estándar de 0.05 , se obtendrían los siguientes resultados (en cifras absolutas): con base en una tasa de crecimiento económico del $6.8 \%$, el número total de trabajadores profesionales y técnicos (grupo 0 en el ISCO) aumentaría de 410000 en 1960 a 715000 en $19 ' 70$ (con margen de error de \pm 25000 ) y a 1240 en 1980 (con margen de error de \pm 80000 ).

Es también posible evaluar las consecuencias que sobre la necesidad de mano de obra altamente calificada tendría una tasa baja de crecimiento económico: para una tasa media anual del $5 \%$ (en lugar

4 Como el incremento en el producto por trabajador se estimó en $3.5 \%$ al año (6.8\% de aumento en el producto menos $3.3 \%$ de aumento en población activa), la proporción de trabajadores profesionales y técnicos aumentaría de $3.5 \times 0.66=$ $2.3 \%$ al año, esto es, $25.5 \%$ en diez años. Así obtenemos: $3.62 \times 1.255=4.54$. 
de $6.8 \%$ establecida anteriormente) la proporción requerida de trabajadores técnicos y profesionales sería de no más del $4 \%$ en 1970 (en vez del $4.65 \%$ ) y de $4.5 \%$ en 1980 (en vez del $5.7 \%$ ). Este ejemplo muestra que las previsiones resultan considerablemente afectadas por la tasa de crecimiento económico, sobre todo si recordamos que estamos hablando de acervos y que las pequeñas variaciones netas en el número de trabajadores técnicos $\mathrm{y}$ profesionales pueden reflejarse en variaciones mayores de las necesidades anuales. En el ejemplo anterior, la diferencia entre las proporciones de tales trabajadores de grado superior de $4.5 \%$ y $5.7 \%$ en 1980 correspondería a una diferencia de 260000 empleos, esto es, a una reducción de un tercio respecto al incremento que se podría esperar en el caso de que la tasa anual de crecimiento económico fuera de $6.8 \% .^{5}$ Es también posible evaluar las consecuencias que en la evolución del grupo de grado superior tendría un incremento más o menos amplio en la productividad del trabajo, especialmente a nivel de sector. Las ecuaciones de regresión harian posible entonces realizar un análisis de sensibilidad

\section{Gráfica 2}

AdMinistradores, GERENTES Y DiRECTORES ( $\mathrm{L} \mathrm{j} / \mathrm{L}$ ) Y PRODUCTO POR TRABAJADOR (X/L) PARA LA ECONOMía EN SU CONJUNTO

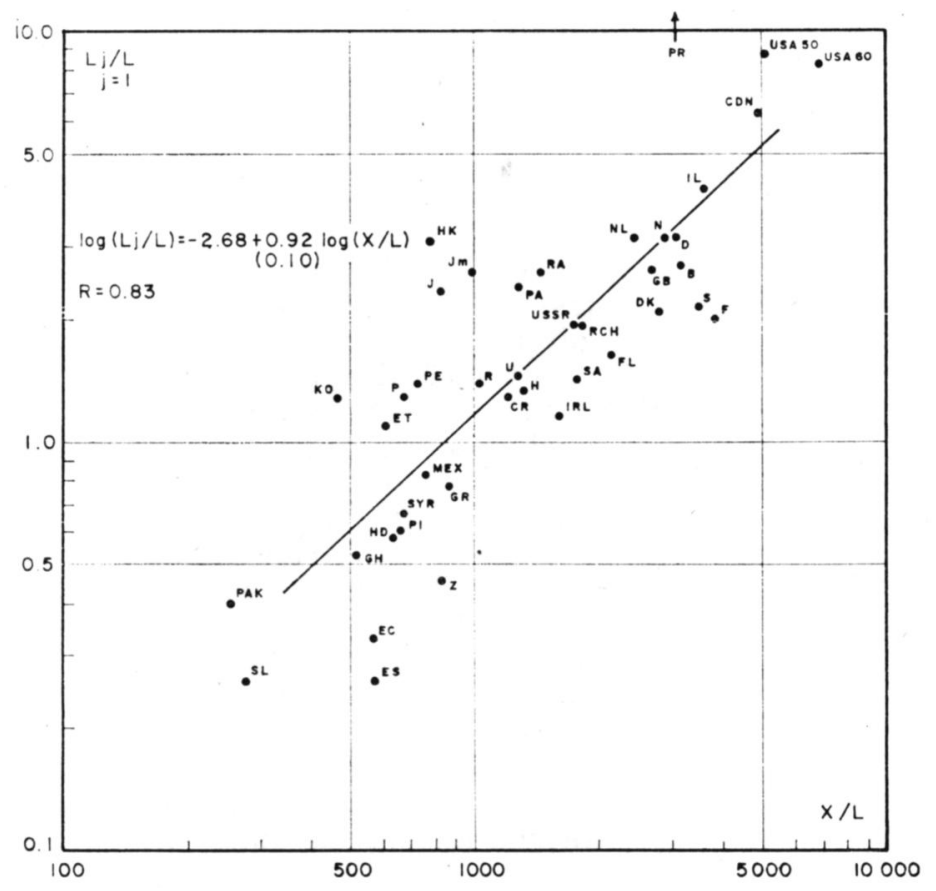

5 Para 1970, la diferencia entre $4.54 \%$ y $4 \%$ sería menor, pero representaría una disminución de $12 \%$ en las cantidades de 1970 , esto es, una diferencia en cifras absolutas de 87000 trabajadores profesionales y técnicos. 
sobre los efectos de las variaciones de los diferentes parámetros o tasas de crecimiento.

En lo que se refiere a la categoría ocupacional "administradores, gerentes y directores" (grupo 1 de la ISCO) cuyo papel en el desarrollo económico es muy importante, de acuerdo con las ideas de Schumpeter concernientes a "empresarios", se pueden hacer observaciones similares: México está cerca de la línea de regresión, pues la proporción de $0.8 \%$ corresponde aproximadamente a un producto por trabajador de Dls. 800 (véase la gráfica 2). La elasticidad de esta categoría ocupacional comparada con la productividad (0.92) es mayor aquí que en el caso precedente y la proporción de gerentes y directores se eleva a 1.1 en 1970 y a 1.5 en 1980 . Pero en este caso la correlación es menor, puesto que el producto por trabajador explica solamente el $69 \%$ de las diferencias en la estructura ocupacional entre los 42 países considerados; como era de esperarse en la dispersión mostrada en la gráfica, la desviación estándar es también mayor que la anterior y los límites de confianza, calculados como antes, varían entre 1 y 1.2 para 1970 y entre 1.3 y 1.8 para 1980 .

Lo anterior también puede expresarse diciendo que el número de "gerentes" (95000 en 1960) debería ser entre 162000 y 193000 en 1970 y entre 264000 y 394000 en 1980.

Es también posible calcular el aumento neto anual ${ }^{6}$ del número de gerentes: sobre la base de las cifras dadas antes, esta tasa estaría entre $6.5 \%$ y $7.35 \%$ al año.

Ahora consideraremos el caso de la industria de transformación tomando en cuenta profesionales y técnicos (grupo 0). Al contrario de los supuestos mencionados anteriormente, la correlación (observada en 36 países) es menor que para la economía en su conjunto: como se muestra en la gráfica 3 , menos de dos tercios de las variaciones en la estructura ocupacional entre países son "explicadas" por el producto por trabajador.

Como el coeficiente de elasticidad es aquí igual a uno, una extrapolación de la tasa de crecimiento industrial de los años recientes (6.9\% al año) correspondería a un aumento similar en el número de profesionales y técnicos, que aumentarán entonces de 47000 en 1960 a 91700 en 1970 y a 179000 en 1980 . Pero en vista del tamaño de la desviación estándar, el margen de incertidumbre sería esta vez de aproximadamente \pm un tercio.

Otra dificultad se origina por el hecho de que México, en la gráfica 3, está localizado muy arriba de la línea de regresión, esto es, la proporción de profesionales y técnicos en ia industria de transformación es mayor ( $3 \%$ ) que en países con un desarrollo industrial comparable en 1960: Grecia (1.5\%), Japón (1.8\%), Perú (1.2\%), Portugal $(0.8 \%)$, etc. Por otra parte, el coeficiente ocupacional de México es comparable con el de aquellos países donde la productividad del trabajo en la industria es mucho mayor: Dinamarca $(2.7 \%)$, Bélgica ( $3 \%)$, etc. Estas observaciones destacan el interés de las comparacio-

6 Lo cual no corresponde al número de empleos nuevos ya que no permite ni el reemplazo de una generación por otra ni los efectos de la movilidad ocupacional. 


\section{Gráfica 3}

INDUSTRIa: Profesionales y técnicos ( $\mathrm{Lij} / \mathrm{Li})$ Y PRODUCTO SECTORIAL POR TRABAJADOR ( $\mathrm{Xi} / \mathrm{Li})$

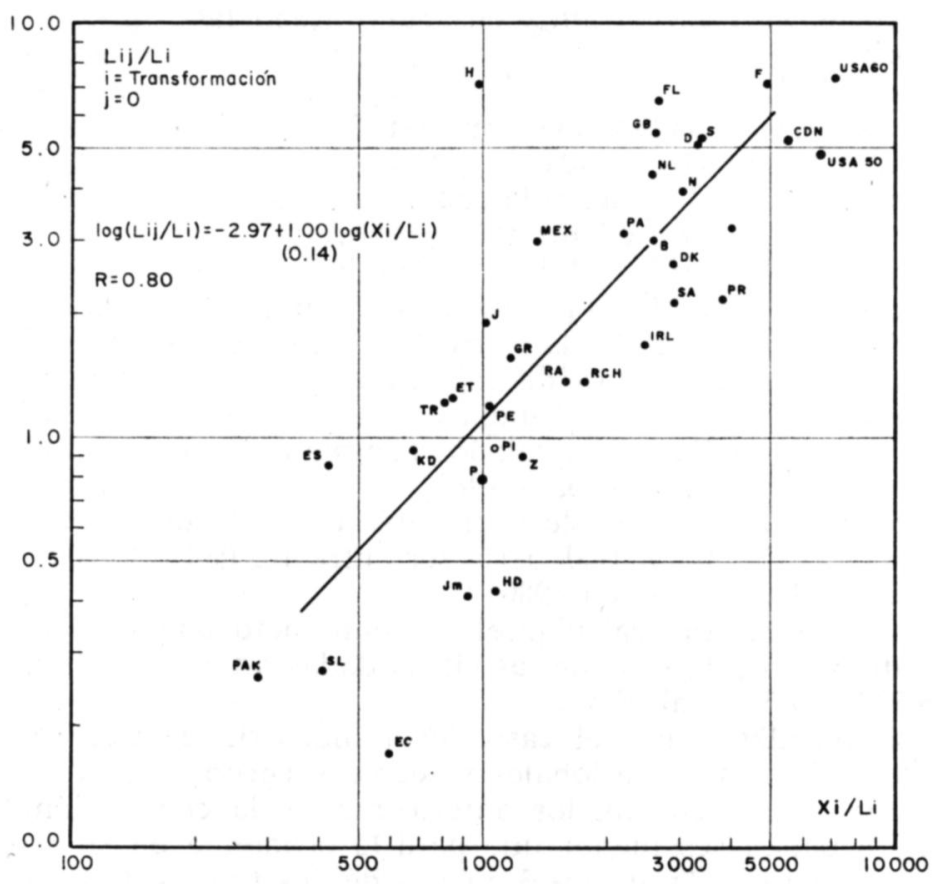

nes internacionales y también lo inadecuado de las mismas. Es ciertamente útil para el planificador poder situar la estructura ocupacional de México en relación a países similares y a países económicamente más avanzados. Pero, ¿cómo deben interpretarse las desviaciones si se comparan con la situación "promedio" mundial, representadas por la línea de regresión? En apariencia, es imposible hacer esto sin recurrir a un criterio de efectividad (o de optimización) que no puede obtenerse de la simple observación de los hechos. El coeficiente de ocupación relativamente alto de México podría interpretarse como indicativo de un "exceso" de personal calificado, exceso que representa la diferencia entre el $3 \%$ observado y el $1.4 \%$ teórico indicado por la ecuación de regresión para una productividad del trabajo valuada en Dls. 1200. Pero el razonamiento opuesto podría también mantenerse suponiendo, por ejemplo, que esta proporción relativamente alta de profesionales y técnicos es uno de los factores explicativos de la tasa de crecimiento de la industria mexicana. Si suponemos que identificamos estos coeficientes ocupacionales con un coeficiente educativo y que una gran proporción de profesionales y técnicos tienen un nivel educativo alto: de acuerdo con la primera interpretación, diríamos que los gastos en educación han sido superiores a las "necesidades" de la economía. En la segunda interpretación, diríamos que la inversión en adiestra- 
miento (de acuerdo con la noción teórica de "capital humano") ha sido un factor de crecimiento. La única manera de esclarecer esta ambiguiedad es recurriendo a un criterio que es externo al modelo presentado antes, esto es, tomando en cuenta las posibilidades de sustitución y la estructura de salarios.

De acuerdo con la teoría económica, la situación especial de México se juzgará óptima o subóptima según represente o no la combinación más eficiente de los distintos factores de la producción, considerando su costo relativo, su elasticidad de sustitución y la contribución respectiva de cada factor a la producción.

En tal contexto es obvio que las necesidades de mano de obra deberían elevarse; pero las dificultades prácticas son muchas, sin hablar de las objeciones teóricas, que principalmente se refieren a las imperfecciones del mercado. Entonces, deberíamos conocer el costo de cada uno de los factores de producción para cada país, cada sector y aún para cada empresa; deberíamos conocer también qué salarios corresponden a cada nivel y tipo de educación y si es posible para cada categoría ocupacional y cada sector económico. También sería necesario tener un criterio adicional para ver si estos salarios reflejan o no una situación óptima en cada país, etc. Por lo tanto, estamos confinados a usar la información disponible para comprobar relaciones simplificadas; aun cuando no tengan un significado teórico satisfactorio, estas relaciones pueden revelar situaciones complementarias cuya medición estadística sea útil a los planificadores para hacer previsiones basadas en la experiencia internacional o simplemente como elemento de juicio. En sentido abstracto, bien podrían refutarse igualmente los argumentos de inspiración neoclásica, que suponen posibilidades casi infinitas de sustitución entre los factores de la producción (ocupaciones, tipos de adiestramiento, etc.) como argumentos en que se basan las funciones parciales de producción mencionadas antes, ya que no podemos suponer que las posibilidades de sustitución no se dan ni que los precios relativos no juegan parte alguna en la operación del mercado de trabajo. El hecho mismo de que todos los países no se encuentren exactamente sobre la línea de regresión sugiere que hay varias combinaciones posibles entre coeficientes educativos, por un lado, y entre los diversos factores de la producción, por otro, que no se toman en cuenta en las ecuaciones que probamos. Desde este punto de vista, la comparación internacional no puede considerarse como un método de previsión para calcular parámetros directamente utilizables para la planeación de las necesidades de mano de obra, pero sí como elemento de juicio sobre el cual pueden hacerse preguntas pertinentes concernientes a la relación entre el sistema educativo y la estructura ocupacional de la economía. El hecho de que tal comparación no muestre ninguna correlación alta desde el punto de vista de ajuste estadístico no debería interpretarse como un fenómeno negativo. Las diferencias entre países que quedan "sin explicación" conducen por el contrario a un análisis más apremiante de la operación del mercado de trabajo.

La gráfica 4, que muestra la relación entre personal de nivel alto (grupos 0 y 1) y el producto por trabajador en la industria de la construcción, puede dar origen a comentarios similares. Cuando estén 
disponibles los resultados de la encuesta que realiza El Colegio de México en asociación con el Centro de Desarrollo de la OCDE sobre las técnicas usadas por las empresas constructoras, será posible ver por qué México tiene mayor proporción de tales trabajadores que los países de nivel económico similar.

Los datos sobre salarios medios por categorías ( $y$ si es posible por niveles y tipos de adiestramiento) deberían hacer posible, entre otras cosas, observar hasta qué punto la alta proporción de los grados

\section{Gráfica 4}

Construcción: Profesionales y administradores ( $\mathrm{Lij} / \mathrm{Li}$ ) Y PRODUCTO SECTORIAL POR TRABAJADOR $(\mathrm{Xi} / \mathrm{Li})$

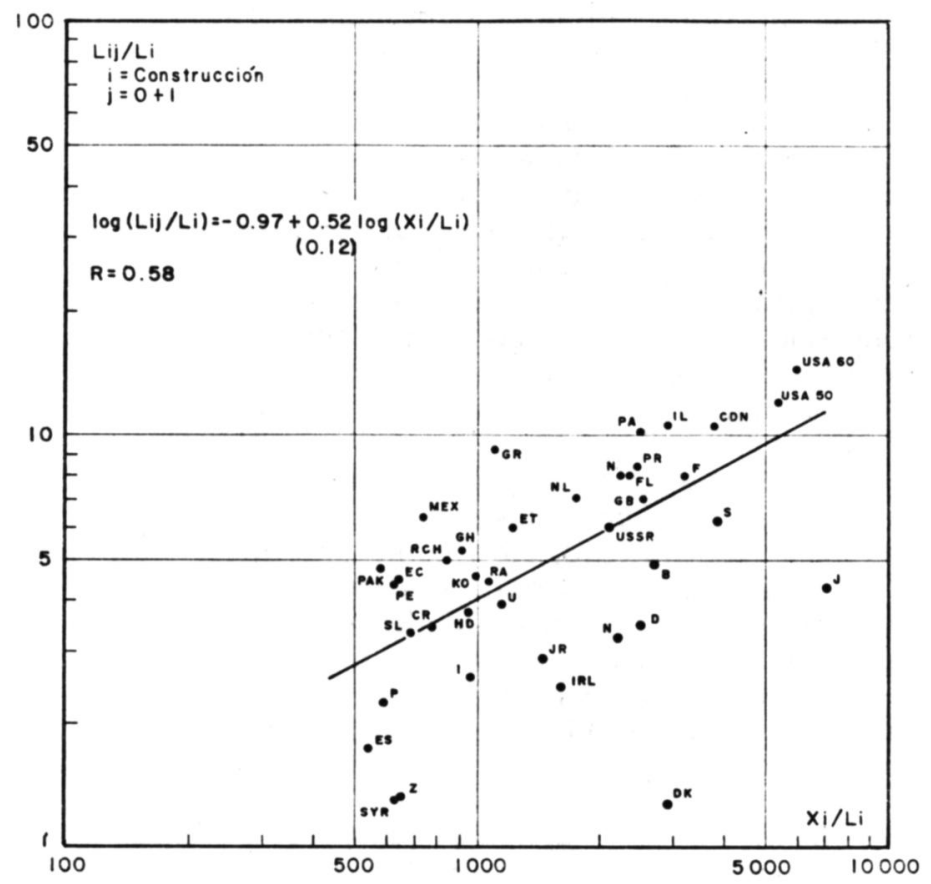

superiores refleja su oferta relativa comparada con otras categorías ocupacionales o comparadas con la disponibilidad de capital, y si ésta es una característica de una industria que hace uso altamente intensivo de personal de nivel alto. Un análisis exhaustivo de estos problemas necesitaría datos desglosados a nivel de empresa (para estudiar la función de producción en cada una) y por trabajador (para poder separar los sueldos y salarios y las respectivas influencias del adiestramiento, categoría ocupacional, edad y otras variables).

Las gráficas 5 a 8 se refieren a la estructura educativa de la población activa.

Por desgracia, los datos disponibles del censo de 1960 de México no proporcionan información acerca de los perfiles educativos de la 
fuerza de trabajo y nos vemos obligados a limitarnos a uno o dos comentarios generales.

La gráfica 5 muestra que no se observa ninguna mejora -muy al contrario- en la relación entre categoría ocupacional y nivel de desarrollo económico cuando se toman en consideración los "perfiles educativos" de las categorías ocupacionales (o, más específicamente, de la proporción de personal de nivel alto con un mínimo de doce años de educación).

\section{Gráfica 5}

NÚMERO DE PROFESIONALES Y TÉCNICOS CON ESCOLARIDAD MEDIA COMO MÍNIMO, COMO PROPORCIÓN DEL NÚMERO TOTAL DE ESTA CATEGORfa $(\mathrm{Ljk} / \mathrm{Lj})$ Y PRODUCTO POR TRABAJADOR $(\mathrm{X} / \mathrm{L})$

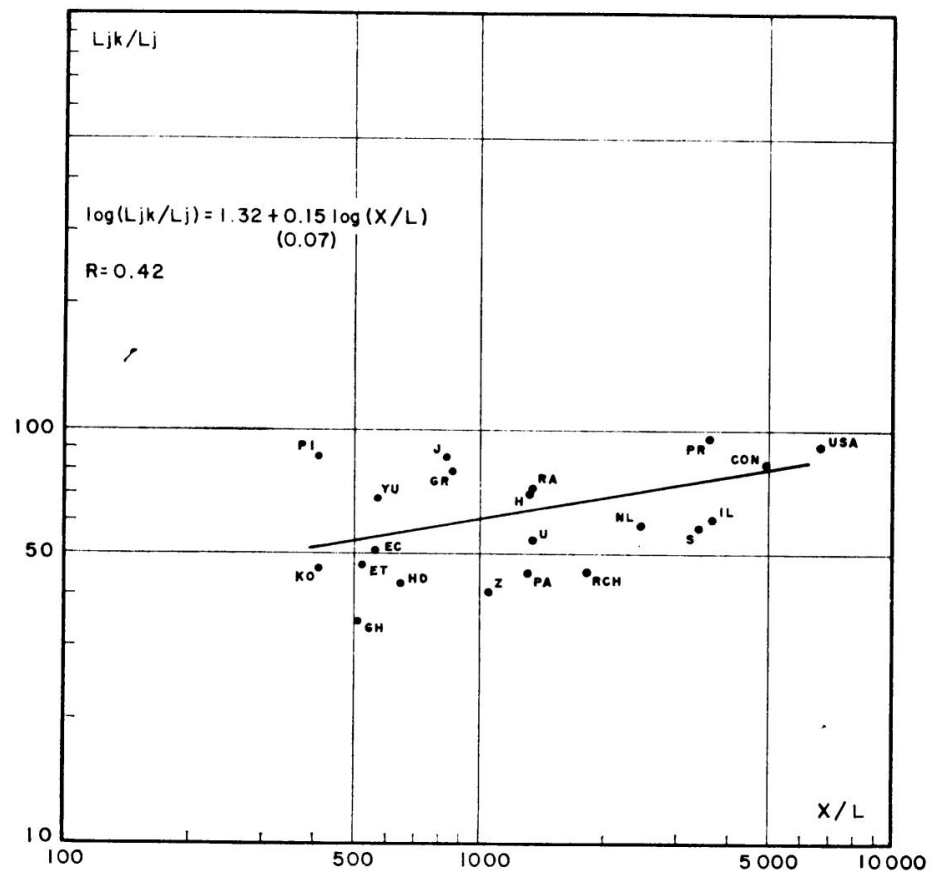

La comparación internacional, por lo tanto, no parece dar validez al procedimiento usado por la mayoría de los planificadores de mano de obra, quienes predicen una elevación en el nivel educativo del personal de nivel alto.

La gráfica 6 muestra el nivel de educación del personal de nivel alto no como porciento de esa categoría sino como un insumo ocupacional y educativo, que significa que solamente se toman en consideración aquellos que poseen un grado universitario: la correlación ( 0.73 ) es mucho más baja que cuando la categoría completa es considerada sin tomar en cuenta el nivel de educación (0.91). No obstante que la eliminación de personal de nivel alto que no ha complementado sus cursos universitarios probablemente mejore la comparabilidad inter- 
nacional de las clasificaciones ocupacionales, no puede decirse que la interpretación económica de la gráfica 1 se confirme.

Un comentario similar puede hacerse con respecto a la gráfica 7 : cuando consideramos una categoría más homogénea que está más directamente relacionada con el desarrollo económico tal como el personal científico y técnico (subgrupos 0-0, 0-1, 0-2 y 0 de la ISCO) con grado universitario, la correlación con el producto por trabajador (0.76) no es mayor que en el caso anterior.

\section{Gráfica 6}

\section{NúMERO DE PROFESIONALES Y TÉCNICOS CON "GRADO UNIVERSitario" COMO PROPORCIÓN DEL EMPLEO TOTAL $(\mathrm{Ljk} / \mathrm{L})$ Y PRODUCTO POR TRABAJADOR (X/L)}

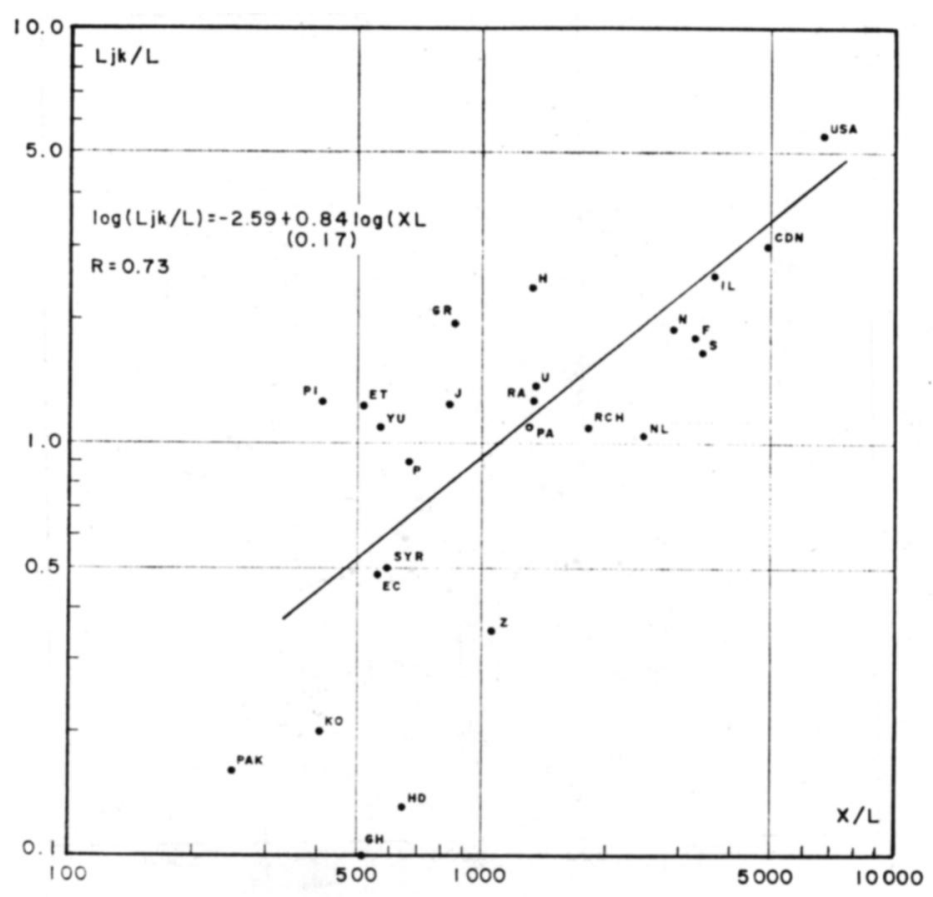

Las necesidades de educación se han previsto algunas veces sin cubrir previamente la etapa de previsión por categorías ocupacionales. El "modelo Correa-Tinbergen" basa su función objetiva en el supuesto de una relación entre el número de graduados activos y el producto nacional. La gráfica 8 muestra que la correlación entre graduados universitarios y el producto por trabajador es muy pequeña (0.65). A diferencia del "modelo Tinbergen", las variables se expresaron en valores per capita para eliminar el efecto de dimensión que reduce considerablemente la significación del modelo referido anteriormente, ya que, por supuesto, es evidente que los países más populosos (como la India) tienen mayor número de graduados que los países industria- 
Gráfica 7
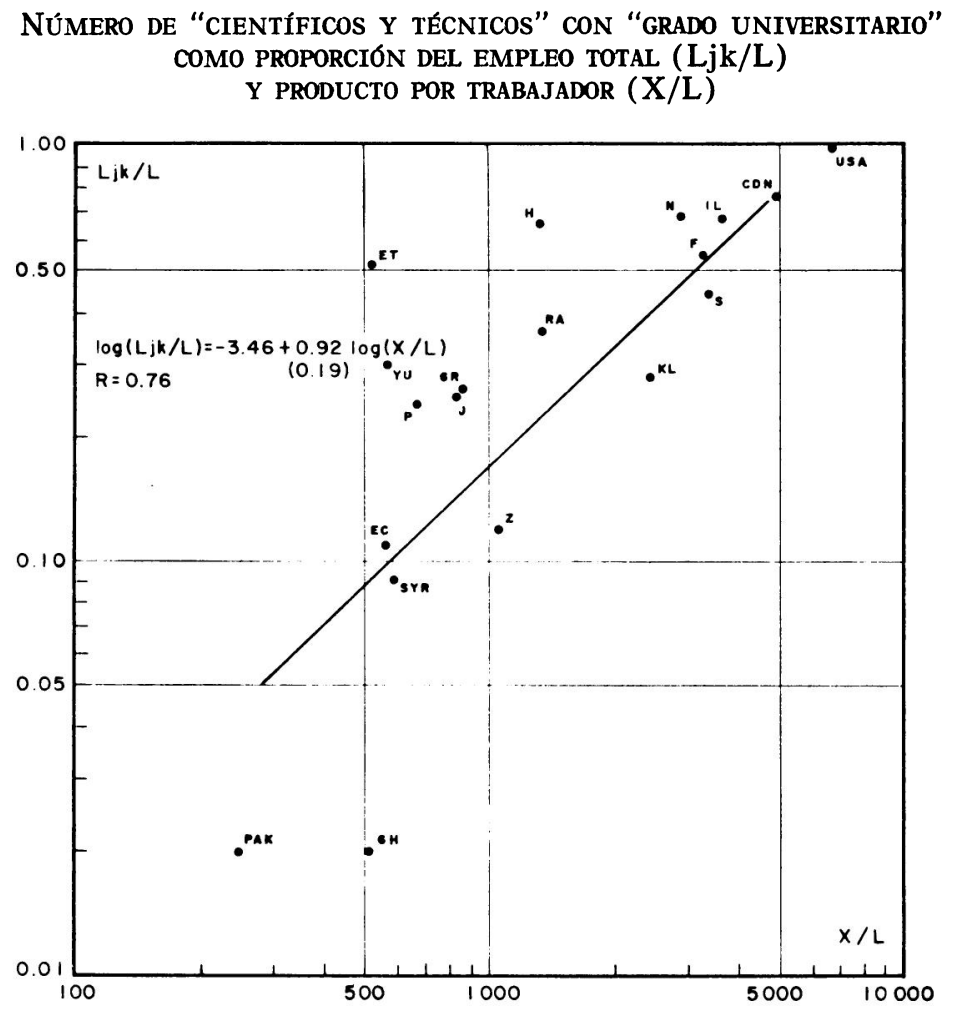

les pequeños (Bélgica, Países Bajos, etc.). Estas relaciones triviales ocultan de hecho las diferencias relativas que aparecen sólo cuando las variables se expresan como función de la población activa de cada país. Considerando los márgenes de incertidumbre de los coeficientes de regresión $(0.38<b<1.1)$, se verá que esta relación no puede usarse para las previsiones de las necesidades de graduados ni para tomar decisiones para aumentar el número de estudiantes en las universidades. La estructura educativa de la población está, por lo tanto, en apariencia, aún menos ligada con el nivel de desarrollo económico que la estructura ocupacional, o una combinación de las dos. Desde un punto de vista práctico, es preferible trabajar a un nivel más desagregado aun si la correlación con los indicadores económicos no es muy alta; en cualquier caso es posible hacer el máximo uso de la información disponible concerniente a las categorías ocupacionales y sectores económicos para una evaluación crítica de la situación actual.

De lo presentado pueden derivarse dos conclusiones que son contradictorias sólo a un nivel de análisis superficial. La comparación internacional es definitivamente de interés para el planificador, especialmente como medio de probar por verificación estadística la ex- 
Gráfica 8

NÚMERO DE PERSONAS CON "GRADO UNIVERSITARIO" COMO PROPORCIÓN DEL EMPLEO TOTAL (Lk/L) Y PRODUCTO POR TRABAJADOR (X/L)

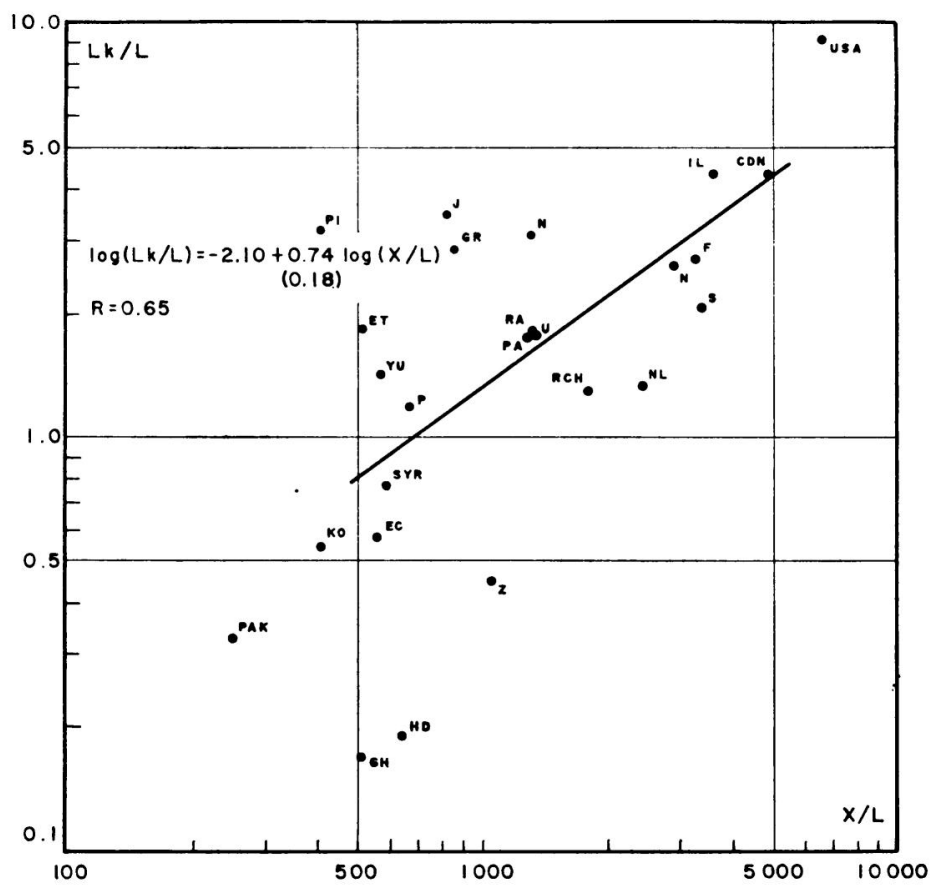

periencia combinada de varios países y el grado de confianza que puede atribuirse a relaciones macroeconómicas simplificadas que están implícitas en la mayoría de los métodos de previsión usados. Se espera a este respecto que los resultados de los censos de 1970 sean analizados sistemáticamente y comparados con los de 1960. Tal vez fuera posible, en particular, ver hasta qué punto la evolución de cada país sigue la tendencia mundial y hacia dónde las relaciones transversales observadas en 1960 han continuado hasta 1970. 